\title{
Measurement of the Beam Normal Single-Spin Asymmetry of ${ }^{12} \mathrm{C}$
}

\author{
Anselm Esser*, Harald Merkel, Michaela Thiel, Sören Schlimme, Concettina Sfienti \\ Johannes Gutenberg-Universität Mainz, Institut für Kernphysik, D-55099 Germany \\ E-mail: esser@uni-mainz.de
}

\begin{abstract}
Beam normal single-spin asymmetry in the elastic scattering of electrons is a direct probe for the two photon exchange. At the Mainz Mictrotron, this asymmetry has been measured for ${ }^{12} \mathrm{C}$ in the $\mathrm{Q}^{2}$ range between 0.02 and $0.05 \mathrm{GeV}^{2} / \mathrm{c}^{2}$. A $570 \mathrm{MeV}$ continuous wave electrons beam was scattered on a carbon target and detected by two magnetic spectrometers. Quartz glass Cherenkov detectors located at the elastic line in the spectrometer's focal plane were used to measure the amount of scattered electrons. The PMTs of which were read out with integrating current ADCs allowing for particle rates too high for counting. The resulting asymmetry shows a surprising deviation from the theoretical predictions.
\end{abstract}

PACS: $25.30 . B f, 27.20 .+n$

54th International Winter Meeting on Nuclear Physics

25-29 January 2016

Bormio, Italy

\footnotetext{
* Speaker.
} 


\section{Introduction}

Over the last decades, parity violating electron scattering (PV) has become an important measurement principle in various fields of hadronic physics. It provides an established method to determine the Weinberg angle, as well as in a more recent experiment, the measurement of the neutron skin of ${ }^{208} \mathrm{~Pb}$ nuclei.

In the case of the Weinberg angle, longitudinally polarised electrons are elastically scattered on a pure hydrogen target. The scattering amplitude is dominated by the electromagnetic interaction, however the neutral weak current poses a non negligible contribution. While the electromagnetic contribution is invariant under parity transformations, which is equivalent to an inversion of the beam helicity, the weak contribution changes its sign under this transformation. This gives rise to a small count rate asymmetry amongst both helicity states. The standard model precisely predicts the Weinberg angle, therefore comparing the measurement results with the predicted value offers a test for physics beyond the standard model. A measurement of this type has been performed e.g. at the Q-Weak experiment [1], another one will be performed by the P2 experiment, which is currently being constructed at the new accelerator facility MESA [2].

The measurement of neutron skins via PV is similar to the Weinberg angle measurements with the difference, that a heavier target nucleus is used. The weak charge of the neutron $\left(Q_{W}^{n} \approx-0.99\right)$ is significantly larger than the one of the proton $\left(Q_{W}^{p} \approx 0.07\right)$, therefore the weak current couples much stronger to the neutron as to the proton. From the PV asymmetry one can then reconstruct the spatial distributions of neutrons in the nucleus. Comparing it to the precisely measured electric form factor, one can then calculate the thickness of the neutron skin, a shell on the surface of the nucleus which is primarily populated by neutrons. The Lead Radius Experiment (PREX) at the Jefferson Lab has for the first time successfully exploited this principle to determine the neutron distribution in ${ }^{208} \mathrm{~Pb}$ nuclei [3]. Similar experiments are planned to be performed at the MESA accelerator.

All measurements of this kind have in common, that they quantify small asymmetries of the order of $10^{-6}$ to $10^{-5}$ and are therefore very sensitive to any form of false asymmetries, i.e. asymmetries which are not caused by parity violation. This implies, that it is of the utmost importance to have a profound understanding of any sources thereof.

\subsection{Beam Normal Spin Asymmetry}

The most significant contribution to false asymmetries is the beam normal spin asymmetry $A_{n}$. It occurs when a non zero polarisation component of the beam exists, which is normal to the scattering plane and changes its sign with the beam helicity. Since no accelerator is perfect, such a component is present in every electron beam.

$A_{n}$ is defined by: $A_{n}=\left(\sigma_{\uparrow}-\sigma_{\downarrow}\right) /\left(\sigma_{\uparrow}+\sigma_{\downarrow}\right)$, where $\sigma_{\uparrow(\downarrow)}$ is the elastic cross sections for the case that the beam spin polarisation is parallel (antiparallel) to a vector $\vec{k}$ which is defined, using the momentum vectors of incident and outgoing electrons $\left(k_{\text {in }}\right.$ and $k_{\text {out }}$ ), by: $\vec{k}=\overrightarrow{k_{\text {in }}} \times \overrightarrow{k_{\text {out }}}$ [4].

The physical cause of the beam normal spin asymmetry is an interference term amongst oneand multi-photon exchange. There are different calculations of $A_{n}$ available, but all of which use a certain set of assumptions. A calculation from Gorchtein and Horowitz [5] takes the full range of intermediate states into account, but is limited to only two photon exchange, also it does not 
model Coulomb distortion effects. Another calculation from Cooper and Horowitz [6] includes all orders of photon exchange, but is limited to only elastic intermediate states. The results of both calculations are not in agreement, still they are used to model the background for PV measurements.

At the Jefferson-Lab the PREX-Collaboration together with HAPPEX performed a measurement of $A_{n}$ for several nuclei $\left({ }^{1} \mathrm{H},{ }^{4} \mathrm{He},{ }^{12} \mathrm{C},{ }^{208} \mathrm{~Pb}\right)$ in the $Q^{2}$ range between 0.01 and $0.1 \mathrm{GeV}^{2} / \mathrm{c}^{2}$. While they found an agreement between their measurement and the theoretical calculations from [5] for light nuclei, for lead the theory predicted a value of $A_{n}^{(t h)} \approx-8 \mathrm{ppm}$, but the measurement resulted in a value of $A_{n}^{(e x)}=0.28 \pm 0.25 \mathrm{ppm}$. This clearly showed, that more investigation in this area or research is necessary.

\section{Measurement at Mainz}

The goal of this experiment was to remeasure the beam normal spin asymmetry of ${ }^{12} \mathrm{C}$ with a high precision in the $\mathrm{Q}^{2}$ range between 0.02 and $0.05 \mathrm{GeV}^{2} / \mathrm{c}^{2}$. This requires an accelerator with a high intensity transverse polarised electron beam of high quality as well as a high rate capable detector system. Additionally, a system of polarimeters for determining the degree of beam polarisation as well as the polarisation orientation is needed.

\subsection{Experimental Set-up}

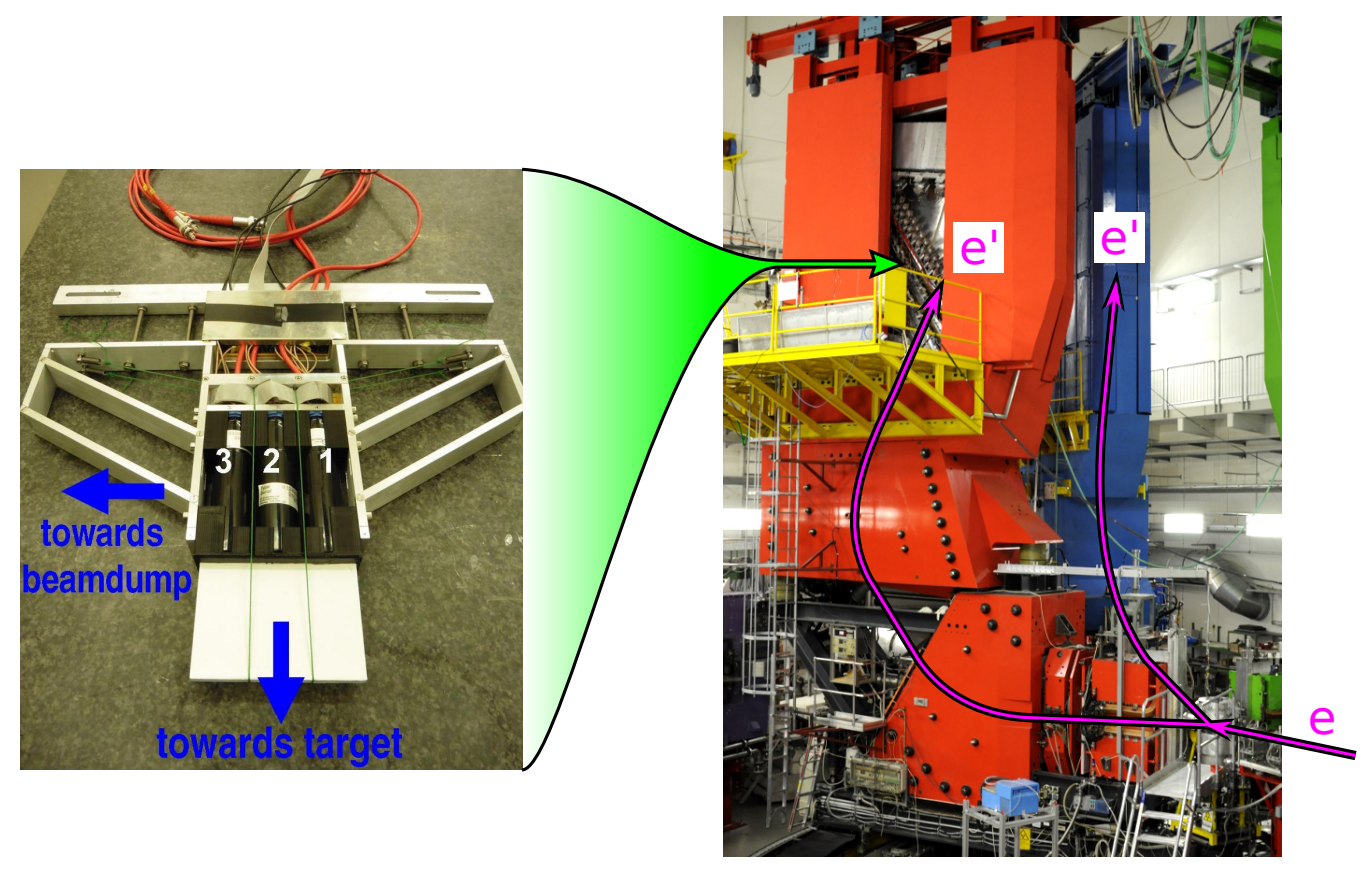

Figure 1: The detectors (left) consist of a Cherenkov radiator (white) coupled to several independently read out PMTs (labelled 1,2 \& 3). They are mounted inside the spectrometers (right) in between the vacuum chamber and the first layer of drift chambers.

The Mainz Microtron (MAMI) is a 5 stage electron accelerator which can deliver polarised and unpolarised continuous wave electron beams with energies between $180 \mathrm{MeV}$ and $1.6 \mathrm{GeV}$. 
One of the experimental facilities is the A1-spectrometer hall with three high-resolution magnetic spectrometers which can be positioned horizontally around a target covering a large range of possible scattering angles [7].

For the measurement an electron beam of $20 \mu \mathrm{A}$ and $570 \mathrm{MeV}$ impinged on a $10 \mathrm{~mm}$ thick carbon target. Detectors, which were specifically designed for this experiment, were placed in the focal plane of the spectrometers, which were placed at forward angles. The spectrometers and one of the detectors are shown in Fig 1. This set-up allowed to make use of the high precision tracking detectors of the spectrometer, to align the elastic line with the additional detectors by changing the magnetic field setting. This was done with a low beam current of approx. $50 \mathrm{nA}$, for the high beam current data taking the tracking detectors were deactivated to protect them from radiation damage.

The focus of the detector design lay on a high detection efficiency combined with a high rate capability. They consist of $10 \mathrm{~mm}$ thick quartz glass Cherenkov radiators coupled to Photomultipliers (PMTs). Since the emitted Cherenkov radiation is in the ultra violet spectral range, the chosen PMTs needed to be equipped with quartz glass windows. Mounting the detectors in the spectrometer limited their over all width to $32 \mathrm{~mm}$, therefore 12 stage 9128QSB PMTs from ET Enterprises were chosen. In order to improve the linearity of the PMTs at high count rates, the voltage dividers were modified to allow for limiting the gain by disconnecting the last dynodes with an arrangement of relays.

The data acquisition electronics used in this experiment were part of the set-up of the A4 Experiment previously conducted at MAMI [13]. Its central component is a gate-generator which provides a measuring gate as well as a series of polarisation states, consisting of patterns " +- + " and "-++-" in a pseudo random sequence, which are used to change the helicity at the beam source. The detector signals and the necessary beam parameters are acquired using ADCs which integrate the charge over periods of $20 \mathrm{~ms}$. This method, in contrast to counting individual events, has the advantage, that it is not subject to pile-up effects and that it is not affected by discriminator thresholds. The $20 \mathrm{~ms}$ integration windows are synchronised to the power grid frequency, thus minimising any effects of ground noise. Additionally to the detector signals, the signals from two $\mathrm{X}$-Y-position monitors, an intensity monitor and an energy monitor as well as a constant current source are acquired in order to reconstruct the beam position, angle, current and energy, and the integration gate length.

\subsection{Polarimetry}

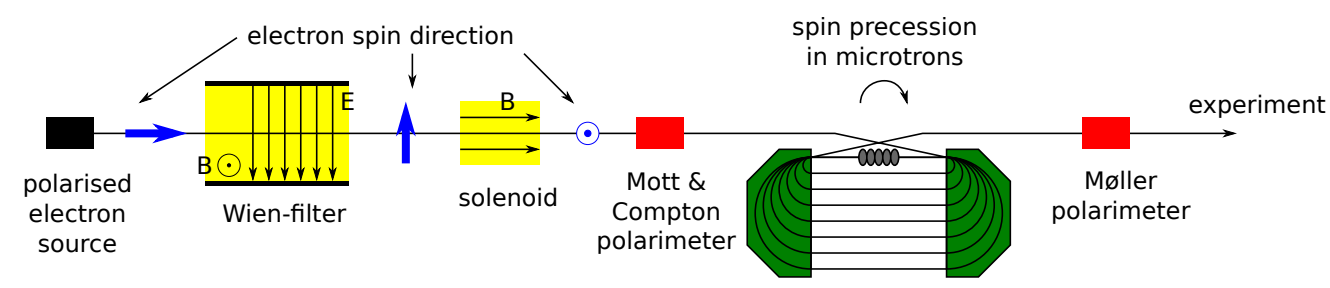

Figure 2: Schematic drawing of all the components required for manipulating and measuring the beam polarisation vector.

Since the MAMI accelerator so far had not been used to provide a vertically polarised beam, it is not equipped with a polarimeter capable of measuring the vertical polarisation component di- 
rectly, but the combination of the existing polarimeters allows for an indirect determination thereof. Fig. 2 depicts the beam-line components used to manipulate and measure the beam polarisation and its direction.

The electron source emits longitudinally polarised electrons with helicities $h= \pm 1$. Next a Wien filter rotates the polarisation around the vertical axis by $90^{\circ}$ to achieve maximum horizontal polarisation. A pair of solenoid magnets, normally used to focus the beam with the fields set to opposite directions, was altered to rotate the polarisation around the beam-line axis to obtain vertical polarisation. At this point two polarimeters of Mott [8] and Compton [9] type enable the measurement of the longitudinal and transverse horizontal polarisation components. Subsequently the electrons are accelerated in a linear accelerator and three stages of race track microtrons to their final energy of $570 \mathrm{MeV}$. Due to the anomalous magnetic moment of the electron the precession of the polarisation vector in the magnetic fields is faster than the momentum change. Therefore a longitudinal polarisation at the electron source is rotated by $55^{\circ}$ counter clockwise at the A1experiment. Just upstream of the experiment a Møeller polarimeter [10] is installed to measure the longitudinal polarisation component at the target.

In order to achieve maximum vertical polarisation, first the Møeller and Mott polarimeters were used independently to measure the absolute degree of polarisation by maximising the longitudinal polarisation at the polarimeters. Subsequently the Wien filter was set to maximise the horizontal transverse polarisation at the source. Both Mott and Compton polarimeters were used to find the optimal setting. In a third step, the solenoid field was set to minimise the horizontal transverse component by rotating the spin vertically. With all the existing polarimeters, it was confirmed, that the remaining horizontal components are in agreement with 0 .

As an additional test, the beam energy was set to $600 \mathrm{MeV}$, thus changing the spin rotation at the target to $139^{\circ}$, and allowing to measure a different horizontal polarisation component using the Møeller polarimeter. This component was also consistent with 0 . Since the depolarisation along the beam line is negligible [11], the previously measured absolute polarisation is in this configuration directed vertically. The procedure of this measurement is described in more detail in [12].

\section{Data Analysis}

As a first step, the data was checked for good events, therefore a small fraction of events was discarded, during which the beam current or the gate-length changed by a noticeable amount. The changes in beam current are mostly related to sudden variations in the beam current stabilisation, while changes in gate-length are caused by power grid frequency fluctuations. Afterwards the asymmetry in the different detector channels is calculated and corrected for beam variations.

\subsection{Correction of False Asymmetries}

Like the PV measurements, this experiment is also subject to false asymmetries caused by variations of the beam which are correlated to the direction of the polarisation. For the purpose of correcting these, the output of several beam monitors are acquired in the same way as the detector signals. From these one can extract the horizontal and vertical position of the beam on the target $(X$ and $Y)$, the two corresponding beam angles $\left(X^{\prime}\right.$ and $\left.Y^{\prime}\right)$ as well as beam current $(I)$ and energy $(E)$. 


\begin{tabular}{|l|l|}
\hline Source of false asymmetry & Correction [ppm] \\
\hline \hline Beam current $(I)$ & -0.83 \\
Beam energy $(E)$ & -0.0090 \\
Horizontal position $(X)$ & 0.10 \\
Vertical position $(Y)$ & -0.00082 \\
Horizontal angle $\left(X^{\prime}\right)$ & 0.010 \\
Vertical angle $\left(Y^{\prime}\right)$ & 0 \\
\hline \hline Further corrections & -4.18 \\
\hline \hline Degree of polarisation & 0.03 \\
Remaining longitudinal polarisation & -0.17 \\
Influence of vertical scattering angle on & \\
beam normal polarisation component & \\
\hline
\end{tabular}

Table 1: List of corrections which are applied on the measured asymmetry for a chosen set-up. The huge correction for the absolute degree of polarisation is caused by dividing the measured asymmetry by the polarisation $(P \approx 82 \%)$

Using the difference of these values between both polarity states, the measured asymmetry is corrected by:

$$
A_{n}=A_{n}^{(m e s s)}-\frac{I^{\uparrow}-I^{\downarrow}}{I^{\uparrow}+I^{\downarrow}}-\left(\Delta X \frac{d \sigma}{d X}\right)-\left(\Delta Y \frac{d \sigma}{d Y}\right)-\left(\Delta X^{\prime} \frac{d \sigma}{d X^{\prime}}\right)-\left(\Delta Y^{\prime} \frac{d \sigma}{d Y^{\prime}}\right)-\left(\Delta E \frac{d \sigma}{d E}\right)
$$

This requires the knowledge of the correction factors, e.g. $d \sigma / d X$. In similar experiments these could be derived from a multilinear regression of the measured asymmetry versus the parameters, however in this experiment the beam was highly stabilised, which excluded this method. Instead the factors were obtained by other means. In the case of the horizontal scattering angle and the energy, the factor was provided by an analytical derivation of a parametrisation of the Mott cross section. For the beam position on the target the factors were obtained by a simulation including the transfer matrices of the spectrometers. The factor for the vertical angle vanishes due to the symmetry in the angular acceptance. However, a vertical scattering angle changes the angle between scattering plane and polarisation direction. Therefore the degree of polarisation is effectively reduced. Subsequently, as a test the factors were compared to factors extracted from a small sample of data which was acquired without beam stabilisations and showed good agreement. A list of the applied corrections is shown in Table: 1

\section{Results}

The preliminary, combined results of both Spectrometers are shown in Fig. 3, together with a theoretical prediction calculated with the model from [5]. The investigation of systematic errors is still in progress, therefore the results are not yet finalised. However the statistical error seems to dominate the overall uncertainty. As can be observed, there is a clear discrepancy between the model and the measurement even for the relatively light ${ }^{12} \mathrm{C}$ nucleus. An explanation for it can be found in the limitations of the theoretical model. On the one hand it only models a two photon 


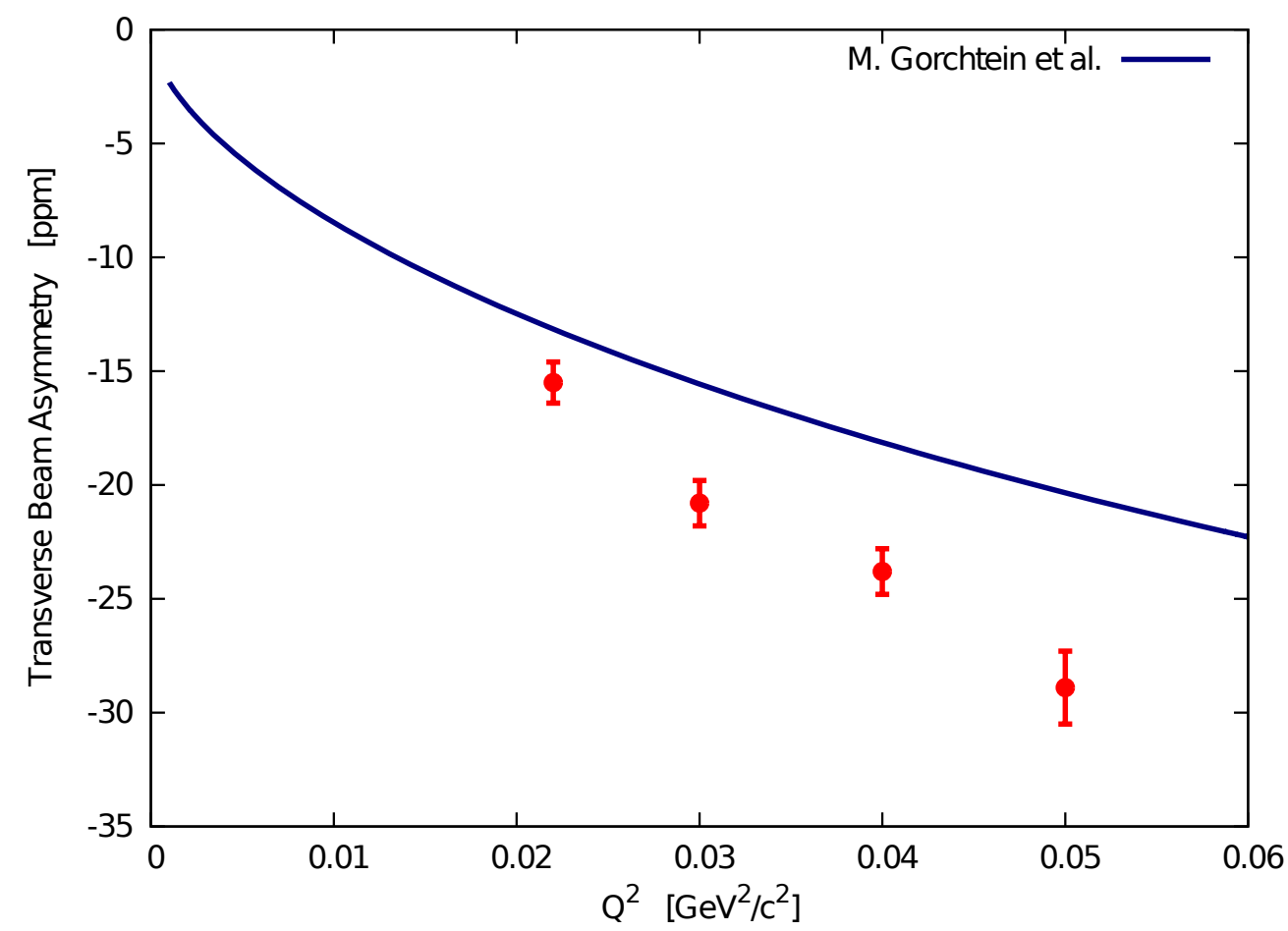

Figure 3: Preliminary results for the measured beam normal asymmetry of ${ }^{12} \mathrm{C}$ (red) and a theoretical prediction based on the model from [5] (blue)

exchange and is technically only valid a scattering angle of $\Theta \approx 0^{\circ}$. On the other hand it does not include coulomb distortion effects and uses a similar nuclear structure for heavier nuclei as it does for hydrogen.

Nevertheless, the calculations of $A_{n}$ are a crucial ingredient for all PV measurements and should therefore be constantly improved. In order to help with this improvement, the upcoming experiments at MAMI will investigate the dependency of $A_{n}$ on the beam energy as well as on the nuclear charge.

\section{Acknowlegements}

This work was supported in part by the Federal State of Rhineland-Palatinate and by the Deutsche Forschungsgemeinschaft with the Collaborative Research Center 1044, as well as by the PRISMA Cluster of Excellence.

We would like to express our gratitude to the team operating the MAMI accelerator, who made this experiment possible by supplying us with an electron beam of highest quality. 


\section{References}

[1] Qweak Collaboration, First Determination of the Weak Charge of the Proton, Phys. Rev. Lett. 111 (2013) 141803

[2] K. Aulenbacher et al., Opportunities for parity violating electron scattering experiments at the planned MESA facility, Hyperfine Interactions 200 (2011) 3

[3] S. Abrahamyan et al., Measurement of the Neutron Radius of ${ }^{208}$ Pb through Parity Violation in Electron Scattering, Phys. Rev. Lett. 108 (2012) 112502

[4] S. Abrahamyan et al., New Measurements of the Transverse Beam Asymmetry for Elastic Electron Scattering from Selected Nuclei, Phys. Rev. Lett. 109 (2012) 192501

[5] M. Gorchtein and C. J. Horowitz, Analyzing power in elastic scattering of electrons off a spin-O target, Phys. Rev. C 77 (2008) 044606

[6] E. D. Cooper and C.J. Horowitz, Vector analyzing power in elastic electron-nucleus scattering, Phys. Rev. C 72 (2005) 034602

[7] K. I. Blomqvist et al., The three-spectrometer facility at the Mainz microtron MAMI, Nucl. Instr. Meth. Phys. Res. A 403 (1998) 263

[8] V. Tioukine et al., A Mott polarimeter operating at MeV electron beam energies, Rev. Sci. Instrum. 82 (2011) 033303

[9] F. Nillius et al., Vector polarimetry at MaMi: Measurements of tensor correlation coefficients in ebremsstrahlungs processes, J.Phys.Conf.Ser. 298 (2011) 012024

[10] P. Bartsch, Aufbau eines Mфller-Polarimeters für die Drei-Spektrometer-Anlage und Messung der Helizitätsasymmetrie in der Reaktion p $\left(e, e^{\prime} p\right) \pi_{0}$ im Bereich der $\Delta$-Resonanz, Ph. D. thesis, Mainz U., Inst f. Kernphysik (2001)

[11] M. Steigerwald, Aufbau einer Quelle polarisierter Elektronen am Injektorlinac und Untersuchungen zur Spindynamik im MAMI. Ph. D. thesis, Mainz U., Inst f. Kernphysik (1998)

[12] B. S. Schlimme, Vertical Beam Polarization at MAMI, in preparation

[13] S. Baunack et al., Measurement of Strange Quark Contributions to the Vector Form Factors of the Proton at $Q^{2}=0.22(\mathrm{GeV} / \mathrm{c})^{2}$, Phys. Rev. Lett. 102 (2009) 151803 